\title{
Evolving phenotype of Marfan's syndrome
}

\author{
Karen J Lipscomb, Jill Clayton-Smith, Rodney Harris
}

\begin{abstract}
Aim-To examine evolution of the physical characteristics of Marfan's syndrome throughout childhood.

Methods-40 children were ascertained during the development of a regional register for Marfan's syndrome. Evolution of the clinical characteristics was determined by repeat evaluation of 10 patients with sporadic Marfan's syndrome and 30 with a family history of the condition. DNA marker studies were used to facilitate diagnosis in those with the familial condition.

Results-Musculoskeletal features predominated and evolved throughout childhood. Gene tracking enabled early diagnosis in children with familial Marfan's syndrome.

Conclusions-These observations may aid the clinical diagnosis of Marfan's syndrome in childhood, especially in those with the sporadic condition. Gene tracking has a role in the early diagnosis of familial Marfan's syndrome, allowing appropriate follow up and preventive care. (Arch Dis Child 1997;76:41-46)
\end{abstract}

Keywords: Marfan's syndrome; evolving phenotype; marker studies.

Marfan's syndrome is an autosomal dominant disorder of connective tissue characterised by musculoskeletal, ocular, and cardiovascular complications. ${ }^{1}$ The underlying molecular defect is caused by mutations in the fibrillin gene on chromosome $15 .^{23}$ Fibrillin, a large glycoprotein, is the principal structural component of the elastin associated connective tissue microfibrils. Microfibrils are particularly abundant in the aorta, periosteum, ciliary muscle zonules, and skin, tissues which are classically involved in Marfan's syndrome. ${ }^{4}$ The phenotype in adults is well documented and in the context of classical multisystem involvement the diagnosis of this condition is relatively straightforward.

Diagnosis of Marfans's syndrome in childhood can be problematic as many manifestations are age dependent and therefore the clinical criteria defined in the Berlin nosology ${ }^{5}$ cannot be strictly applied (table 1). This finding, combined with the extreme phenotypic heterogeneity both between different families and within individually affected family members and with the high incidence of sporadic cases $(20-30 \%)$, contributes to the diagnostic difficulty. ${ }^{1}$ Mutation analysis of the fibrillin gene on chromosome 15 is presently unhelpful as mutations have not been found in
Table 1 Diagnostic criteria for Marfan's syndrome according to the Berlin nosology 1986

\begin{tabular}{cl}
\hline Cardiovascular & $\begin{array}{l}\text { Dilation of ascending aorta } \\
\text { Aortic dissection }\end{array}$ \\
& $\begin{array}{l}\text { Mitral regurgitation } \\
\text { Mitral valve prolapse } \\
\text { Abdominal aortic aneurysm } \\
\text { Ectopia lentis }\end{array}$ \\
Severe myopia \\
Retinal detachment, flat cornea, and \\
elongated globe \\
Dural ectasia
\end{tabular}

In the absence of a definitely affected first degree relative, there should be skeletal involvement plus involvement of two other systems, one with a major manifestation. ${ }^{\star}$ Two system involvement with one major manifestation is required when a first degree relative is affected.

most families, and where they have been defined each kindred seems to have a unique mutation. Gene tracking with a panel of highly informative intragenic microsatellite markers has been established as a useful tool in the presymptomatic diagnosis of familial Marfan's syndrome, allowing appropriate follow up and preventive care. ${ }^{6-8}$ Previous studies documenting the clinical characteristics of adults and children with severe cardiac abnormalities have given little appreciation of the extracardiac morbidity and the mode and age of presentation in childhood. ${ }^{9-16}$

We present the clinical features of 40 children under the age of 16 years in whom early diagnosis was made by clinical and molecular techniques. The evolution of the phenotypic features is described and may assist in the clinical diagnosis of Marfan's syndrome.

\section{Patients and methods}

Patients were ascertained during the development of a regional register for Marfan's syndrome, which was established with the aim of reducing premature death from cardiac complications of this condition. Probands were identified by reviewing case notes in the departments of genetics and cardiology. Diagnosis was confirmed in the proband, which in some cases was the child, and subsequently all first degree relatives at $50 \%$ risk of this autosomal dominant condition were screened by physical examination, echocardiography, and ophthalmological examination. Forty children under the age of 16 years were affected during the course of the study. Individuals 
Table 2 Patient characteristics

\begin{tabular}{|c|c|c|c|c|c|c|c|c|c|c|c|c|c|c|c|c|c|c|}
\hline $\begin{array}{l}\text { Patient } \\
\text { No }\end{array}$ & $F H / S P$ & $\begin{array}{l}\text { Age at } \\
\text { diagnosis } \\
\text { (years) }\end{array}$ & $\begin{array}{l}\text { Presenting } \\
\text { feature }\end{array}$ & $\begin{array}{l}\text { Height } \\
\text { centile }\end{array}$ & $\begin{array}{l}\text { Weight } \\
\text { centile }\end{array}$ & Pal & $A R$ & Sco & $P D$ & $P P$ & $S T$ & $F F$ & Dis & $E L$ & $M Y$ & $M V P$ & $M R$ & $A D$ \\
\hline 1 & $\mathrm{FH}$ & 16 & MS, cardiac & 90th & 50th & + & + & + & + & - & - & + & + & - & - & - & - & + \\
\hline 2 & $\mathrm{FH}$ & 14 & MS & 97th & 50th & + & + & + & - & + & - & + & + & - & - & - & - & - \\
\hline 3 & SP & 7 & MS & $>97 \mathrm{th}$ & 50th & + & + & + & + & + & + & + & + & - & - & - & - & + \\
\hline 4 & FH & 2.5 & MS & $>97$ th & 50th & + & + & + & + & + & - & + & + & - & - & - & - & - \\
\hline 5 & SP & 16 & MS & $>97$ th & 75th & - & + & - & + & + & + & + & + & - & - & + & + & - \\
\hline 6 & $\mathrm{FH}$ & 11 & MS & $>97$ th & 50th & + & + & + & + & + & + & + & + & - & - & - & - & + \\
\hline 7 & $\mathrm{FH}$ & 9 & MS, cardiac & $>97$ th & 50th & + & + & - & + & + & - & + & + & - & - & - & - & + \\
\hline 8 & $\mathrm{FH}$ & 0.5 & MS & $>97$ th & 50th & + & + & - & + & + & - & + & + & - & - & - & - & + \\
\hline 9 & SP & 14 & MS, cardiac & $>97$ th & 75th & + & + & + & + & - & - & + & + & - & - & - & - & + \\
\hline 10 & FH & 2 & MS & $>97$ th & 97th & + & + & - & + & + & - & + & + & - & + & - & - & - \\
\hline 11 & $\mathrm{FH}$ & 3 & MS & $>97$ th & 97th & + & + & - & + & + & - & + & + & - & - & - & - & - \\
\hline 12 & SP & 3 & MS, ocular & $>97$ th & 97th & + & + & + & - & + & + & + & + & + & + & - & + & + \\
\hline 13 & $\mathrm{FH}$ & 15.5 & MS & $>97$ th & 50th & + & + & + & + & - & + & + & + & - & - & - & - & + \\
\hline 14 & FH & 13.5 & MS & $>97$ th & 75th & + & - & - & - & - & + & + & + & - & - & - & - & - \\
\hline 15 & FH & 13.5 & MS & $>97$ th & 50th & + & + & - & + & - & + & + & + & - & - & - & - & - \\
\hline 16 & $\mathrm{FH}$ & 10 & MS & $>97 \mathrm{th}$ & 25 th & + & - & + & + & + & - & + & + & - & - & + & + & - \\
\hline 17 & $\mathrm{FH}$ & 12.5 & MS & $>97$ th & 75th & + & + & + & + & + & + & - & + & - & - & - & - & - \\
\hline 18 & $\mathrm{FH}$ & 11 & MS & $>97$ th & 75th & + & - & + & - & - & - & - & + & - & - & + & + & - \\
\hline 19 & SP & 14 & MS & $>97$ th & 50th & + & + & + & + & + & + & + & + & - & + & - & - & - \\
\hline 20 & $\mathrm{FH}$ & 10 & MS & 75th & 50th & - & + & + & + & + & + & - & + & - & - & - & - & - \\
\hline 21 & SP & 14 & MS & $>97$ th & 75th & + & + & + & + & + & + & + & + & - & - & - & - & - \\
\hline 22 & SP & 11 & MS, cardiac & $>97$ th & 75th & - & + & + & + & + & + & + & + & - & - & - & - & + \\
\hline 23 & SP & 11 & MS & 25 th & 3 rd & + & + & + & + & + & + & + & + & - & + & - & - & - \\
\hline 24 & $\mathrm{FH}$ & 1.5 & Ocular & 90th & 25th & + & + & + & - & - & + & + & + & + & + & - & - & + \\
\hline 25 & FH & 4 & MS & 97th & 75th & + & + & + & + & + & - & - & + & - & - & - & - & + \\
\hline 26 & $\mathrm{FH}$ & 2.5 & MS & 75th & 10th & + & + & - & + & + & + & - & + & - & - & - & - & - \\
\hline 27 & $\mathrm{FH}$ & 7 & MS & 97th & 50th & + & + & - & - & + & - & + & + & - & - & - & - & + \\
\hline 28 & FH & 5 & MS & 75th & 50th & + & + & + & + & + & - & + & + & - & - & - & - & - \\
\hline 29 & $\mathrm{FH}$ & 12 & MS, cardiac & 90th & 50th & + & + & - & - & + & - & + & + & - & - & - & - & + \\
\hline 30 & $\mathrm{FH}$ & 4 & MS & $>97$ th & 50th & + & + & + & + & + & + & + & + & - & + & - & - & + \\
\hline 31 & $\mathrm{FH}$ & 4 & MS & $>97$ th & 25 th & + & + & + & + & + & + & + & + & - & - & - & - & + \\
\hline 32 & $\mathrm{FH}$ & 3 & MS, ocular & $>97$ th & 50th & + & + & - & + & + & - & + & + & + & + & - & - & - \\
\hline 33 & $\mathrm{FH}$ & 4 & MS, ocular & 97th & 75th & + & + & - & + & + & + & + & + & + & - & - & - & - \\
\hline 34 & $\mathrm{FH}$ & 0.25 & MS & $>97$ th & 97th & - & + & + & - & + & - & - & - & - & - & - & - & - \\
\hline 35 & FH & 14 & Ocular & 90th & 25 th & + & + & + & - & + & + & + & + & + & + & - & - & + \\
\hline 36 & FH & 2 & MS, ocular & $>97 \mathrm{th}$ & 50th & + & + & + & + & + & - & + & + & + & + & + & + & + \\
\hline 37 & $\mathrm{FH}$ & 12 & MS & $>97$ th & 50th & + & + & + & + & + & + & + & + & - & - & + & + & - \\
\hline 38 & SP & 10 & MS & $>97$ th & 50th & + & + & + & + & + & + & + & + & - & + & + & - & - \\
\hline 39 & SP & 14 & MS, cardiac & $>97$ th & 50th & + & + & + & + & - & + & + & + & - & + & + & + & - \\
\hline 40 & FH & 0.25 & MS & $>97$ th & 50th & + & + & - & + & - & - & - & + & - & - & - & - & - \\
\hline
\end{tabular}

FH, family history; SP, sporadic case; MS, musculoskeletal; Pal, high arched palate; AR, arachnodactyly; Sco, scoliosis; PD, pectus deformity; PP, pes planus; ST, striae; FF, facial features; Dis, limb disproportion; EL, ectopia lentis; MY, severe myopia; MVP, mitral valve prolapse; MR, mitral regurgitation; AD, aortic dilatation; +, present; -, absent.

reported here were examined on a minimum of two occasions by the authors and many were seen annually for up to 10 years. Neonates with Marfan's syndrome were not included as they have a different prognosis and clinical picture. ${ }^{17}$ All were evaluated by detailed physical examination, ophthalmological examination, and echocardiography. A full family pedigree was obtained and DNA marker studies carried out where appropriate.

Gene tracking was carried out with DNA extracted from peripheral blood. Amplification of polymorphic intragenic markers for the fibrillin gene on chromosome 15 was performed using a polymerase chain reaction. Gene tracking was used only where two or more unequivocally affected individuals were identified within a family.

\section{Results}

A diagnosis of Marfan's syndrome was established in 40 children ( 26 boys and 14 girls) from 29 kindreds. Ten children had sporadic Marfan's syndrome and 30 a clear family history of the condition. The mean (SD) age at diagnosis in patients with a sporadic syndrome (11.4 (3.95) years) was greater than for those with a familial condition ( 7.31 ( 5.23 ) years). In the absence of an unequivocally affected first degree relative, the requirements for diagnosis according to the Berlin criteria are stricter and therefore children with sporadic Marfan's syn- drome will tend to be more severely affected and inevitably age at diagnosis will be greater than for those with a familial condition. Table 2 summarises the details.

\section{MUSCULOSKELETAL CHARACTERISTICS}

The most notable feature at the time of presentation was height equal to or greater than the 97th centile. One child with sporadic Marfan's syndrome did not fulfil this finding having a height on the 25 th centile. In this child height was in excess of that predicted by parental heights and therefore still fulfilled the criteria of being tall compared with that of first degree relatives. Two children have received treatment to limit eventual height. Treatment with testosterone was initiated at the age of 14 years in a boy who had attained a height of $197 \mathrm{~cm}$ (>97th centile) with no radiological evidence of epiphyseal fusion. An 11 year old girl having reached a height of $178 \mathrm{~cm}$ ( $>97$ th centile) was treated with ethinyl oestradiol for four years and subsequently attained a height of $189 \mathrm{~cm}$ ( $>97$ th centile). Interpretation of the upper segment to lower segment ratio to assess limb disproportion in childhood is difficult as the ratio tends to decrease with age, ${ }^{19}$ however, fig 1 shows that the ratio declined within the abnormally low range in most patients. Thirty seven individuals had an exaggerated arm span exceeding height by at least $5 \mathrm{~cm}$. Throughout childhood and adolescence, in contrast to 


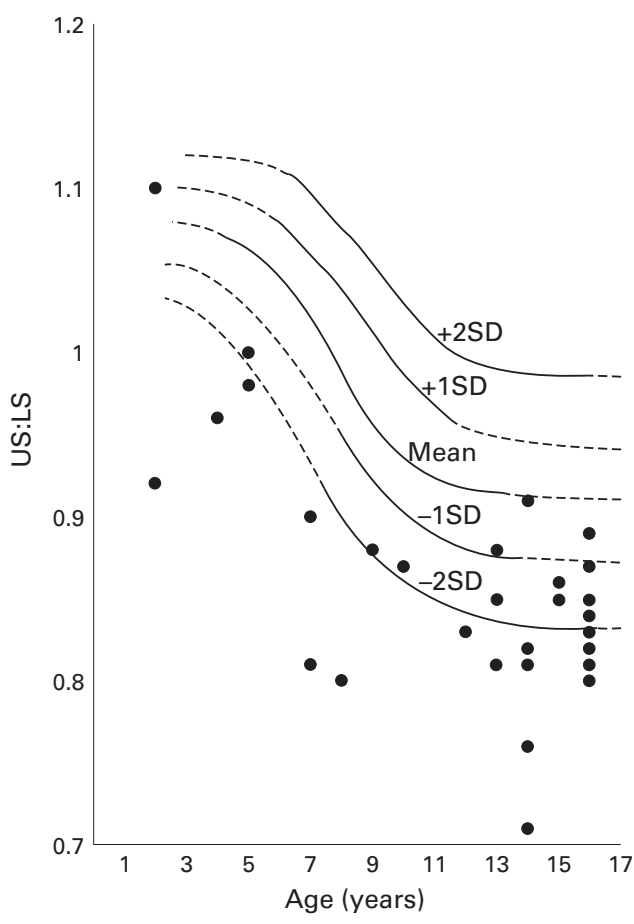

Figure1 Upper segment : lower segment ratio in Marfan's syndrome.

height, the median weight at the time of diagnosis and on subsequent assessment was about the 50th centile.

Thirty one children had a chest deformity and the youngest age at which this was detected was 18 months. A pectus carinatum deformity was documented in 17 patients and in eight of these there was marked asymmetry, while the excavatum deformity noted in 14 individuals tended to be symmetrical. Abnormality of chest shape was noted before the age of 10 years in all individuals but the deformity often worsened during adolescence. Only two had surgical correction of an excavatum deformity which had progressed rapidly before the age of 10 years.

A scoliosis was present in 27 children. The youngest age at which this was noted was 14 months. The affected parent of this child had severe scoliosis in childhood and underwent spinal fixation with Harrington rods. Twenty three individuals with mild scolioses remain under observation. Four children have been treated successfully with physiotherapy, none has required bracing or Harrington rod insertion. Only two had symptomatic back pain. Back pain in adults with Marfan's syndrome is a common complaint, which suggests that although the scolioses were mild in most individiuals, they account for considerable morbidity in later life.

The presence of arachnodactyly was defined clinically by a positive Steinberg sign when the thumb protrudes from the ulnar border of the hand when apposed across the palm ${ }^{20}$ and a positive Walker-Murdoch sign when a combination of slim wrists and long digits results in noticeable overlap of the thumb and little finger when the wrist is grasped. ${ }^{21}$ This was found in all except three children and was identifiable from birth in five.

Thirty one children had pes planus which in most was associated with joint hypermobility with a Beighton score of greater than $5 / 9 .^{22}$ The most common symptom of joint hypermobility, in addition to finger flexibility, was a tendency for the ankle to turn over easily. This may have contributed to delayed age at walking (greater than 18 months) in eight patients who responded to ankle support boots. Joint dislocation was seen in only three children and involved dislocation of the patella.

A narrow, high arched palate was seen in 36 of 40 individuals. This usually became evident around the age of three to four years. Dental crowding necessitating extraction and dental bracing was a frequent feature in adolescents, the timing of intervention being dependent on the age of the patient and eruption of secondary dentition.

The classical facial features of Marfan's syndrome with mid-face hypoplasia, micrognathia, and down sloping palpebral fissures were noted in 33 children.

The presence of multiple (more than six) red striae distensae unrelated to weight gain over the lumbar region and shoulders is a feature common in many adults with Marfan's syndrome. Although striae were found in $88 \%$ of children in the study who were followed up to the age of 16 years, they were noted in only one child before the age of 11 years. This feature may manifest about the time of puberty as rapid growth spurts earlier in childhood do not seem to be associated with development of stretch marks.

Pneumothorax did not occur in any individual in our study. Three children are asthmatic which is not significantly different from the incidence of asthma in the general population.

\section{OCULAR MANIFESTATIONS}

Ectopia lentis was found in only six children $(15 \%)$. This percentage corresponds to the incidence of ectopia lentis in adults with Marfan's syndrome in our study. No patient had lens dislocation before the age of 18 months. Five children with ectopia lentis represent four families in which some, but not all, other affected individuals have lens dislocation. Eleven individuals had severe myopia (greater than 5 dioptres), all diagnosed before the age of 10 years. Two are registered as partially sighted and receive educational support for visual disability. No child experienced retinal detachment.

\section{CARDIOVASCULAR MANIFESTATIONS}

Twenty two patients had echocardiographic evidence of cardiovascular abnormality. All were asymptomatic. Aortic root dilatation, defined in relation to body surface area by standard graphs, ${ }^{23}$ was shown echocardiographically in 17 patients. This was associated with mild aortic incompetence in one patient. The widest aortic root diameter recorded was $4.5 \mathrm{~cm}$ in a 15 year old girl. No individual less than 16 years of age had an aortic dissection. 
Major aortic involvement, defined as aortic dissection or need for elective aortic surgery, was documented in 14 of 19 families represented by the 30 children with familial Marfan's syndrome in this study group. Mitral valve prolapse was documented in seven patients, six of whom had associated mitral regurgitation.

EVOLUTION OF THE PHENOTYPE

Neonatal period

No major problems were encountered in the neonatal period. Birth weights were all within two 2 SDs of the mean and did not cluster in the lower range of normal. Birth length was not recorded in most patients. Arachnodactyly was noted in five patients at birth.

\section{Psychomotor development}

Age of walking was delayed in eight children who had profound joint hypermobility and ankle laxity. No delay in speech development was reported. Three children have mild learning disability, which may be due to visual impairment in one. This does not differ significantly from the incidence of learning deficit in the general population.

\section{Natural history}

The most common presenting feature was musculoskeletal abnormality in the form of tall stature. This represented a marked deviation from the height of unaffected siblings. Height in excess of the 97th centile was apparent in most affected children by the age of 2 years and thereafter the increase in height paralleled normal velocity curves but remained above the 97th centile. A scoliosis became apparent in most patients between the ages of 10 and 14 years (mean (SD) $10.3(4.4)$ ), although it was evident before the age of 6 years in six patients. The mean (SD) age at which chest wall deformity was manifest was 6.9 (3.3) years. Classical facial features together with a narrow, high arched palate was usually evident about the age of 3 to 4 years. Two of 18 children followed to the age of 16 years did not develop striae distensae. The mean (SD) age at which striae were first noted was 13.9 (1.9) years. The presence of pes planus occurred in most patients from about the age of 2-3 years.

Ocular abnormality in the form of ectopia lentis was the presenting feature in six children and was noted between the ages of 18 months and 14 years. No child presented with lens dislocation above this age. Significant myopia was detected in 11 children between the ages of 4 and 10 years.

The mean (SD) age at which aortic root dilatation was detected echocardiographically was 11.0 (3.9) years. In two unrelated familial children there were no other family members with major aortic involvement. The remaining 11 familial children all had a family history of major aortic involvement. The earliest age at which aortic root dilatation was documented was 18 months. The affected parent of this child had an aortic dissection at the age of 17 years.

\section{DNA marker studies}

In 10 families, intragenic polymorphic markers allowed the particular copy of the fibrillin gene that co-segregated with Marfan's syndrome to be identified in affected family members. Eighteen children who were at risk from these 10 families were tested and found to carry this copy of the fibrillin gene, suggesting that they had inherited the condition. In 12 children with familial Marfan's syndrome from nine different kindreds it was not possible to perform gene tracking as there were inadequate numbers of unequivocally affected individuals in previous generations. In these small families in which few meioses are available for scoring, there is potential for error as genetic heterogeneity has been reported by one group of investigators. ${ }^{24}$

\section{Discussion}

Previous reports of children with Marfan's syndrome have concentrated on severely affected individuals with prominent cardiovascular involvement and have included patients with the more severe sporadic neonatal form of this condition..$^{9-12} 17$ Neonatal Marfan's syndrome has a poor prognosis with death from cardiovascular involvement occurring usually in the first year of life. ${ }^{18}$ All reported cases to date have been sporadic, some of which have been due to new dominant mutations clustering in the same region of the fibrillin gene. ${ }^{25} 26$ As such, neonatal Marfan's syndrome is a separate condition and it has been excluded from this study. The clinical features of 40 patients with classical Marfan's syndrome under the age of 16 years are presented here. Thirty patients had a family history of the syndrome and were therefore screened to make an early diagnosis and institute appropriate follow up and preventive care. The identification of two or more unequivocally affected individuals within a family has enabled the use of a gene tracking technique to confirm clinical diagnosis in a child with a $50 \%$ risk of the condition in whom evolution of the phenotype is incomplete at initial assessment. This allows appropriate ophthalmic, cardiac, and orthopaedic follow up and intervention to be planned and has enabled observation of the development of the phenotypic characteristics with increasing age.

The most frequent musculoskeletal findings at presentation were a combination of tall stature ( $>97$ th centile), limb disproportion, pectus deformity, classical facial features, high arched palate, and arachnodactyly. A pectus deformity and development of a scoliosis often became more prominent during the pubertal growth spurt, at which time many children developed noticeable red striae distensae over the lumbar region and shoulders. A minimum of four skeletal features was present in each patient at the time of last evaluation.

Severe cardiovascular abnormalities were not documented as frequently suggested in previous studies. ${ }^{9-1214}$ This possibly may be explained by the exclusion of neonatal patients with Marfan's syndrome. Some $55 \%$ of patients less than 16 years of age had 
cardiovascular involvement but remained asymptomatic. Echocardiographic findings of mild aortic root dilatation were found in 17 patients, usually becoming evident about the age of 9-15 years. This suggests that the absence of aortic root enlargement cannot be used to exclude a diagnosis of Marfan's syndrome in a 'marfanoid' child before skeletal growth is complete. A previous study of serial measurements of the aortic root diameter in children with Marfan's syndrome suggested that the aortic root diameter was at or just above the upper limits of normal throughout childhood. In most individuals serial measurements demonstrated that growth velocity of the aortic root resembles that in normal individuals ${ }^{14}$; however, an increase in growth velocity of the aorta may signify increasing likelihood of aortic dissection or rupture. Although this is rare in childhood or adolescence, it is well documented in the late teens and early twenties. Annual echocardiographic assessment is essential throughout childhood to detect early aortic root dilatation and follow the rate of change of aortic root diameter. Not all patients who satisfy the diagnostic criteria for Marfan's syndrome are at risk of severe cardiovascular complications and a reliable assessment of cardiovascular risk cannot be safely made on the basis of events in preceding generations. ${ }^{27}$

Ophthalmological assessment in our study found that lens dislocation and severe myopia occurred in only about $25 \%$ of patients with Marfan's syndrome. A previous study prospectively evaluated 62 children with Marfan's syndrome who were diagnosed before the age of 5 years and suggested that the mean (range) age of lens dislocation was 2.7 years with a range of 2 months to 6 years of age. ${ }^{28}{ }^{29}$ This suggests that repeat ocular examination is necessary in early childhood to detect lens dislocation but that myopia is likely to be the main complication after the age of about 6 years. Attention must be paid to correction of acuity in early childhood to prevent development of amblyopia.

Diagnosis in patients with familial Marfan's syndrome is confounded by considerable intrafamilial phenotypic heterogeneity. From the families represented in this study severe aortic complications clearly do not affect all individuals within a given pedigree and the age at which aortic events occur within a family is also widely variable. Likewise ocular features do not necessarily 'breed true'. Musculoskeletal characteristics also show intrafamilial variability but to a lesser extent. As with the sporadic syndrome, the phenotype will evolve with age. Gene tracking is useful to provide an early diagnosis when no or minimal skeletal characteristics are evident at the first referral in childhood. It is also of benefit in excluding the diagnosis in a child with a $50 \%$ risk of Marfan's syndrome.

Diagnosis of Marfan's syndrome in a child with no family history of the condition is more difficult and diagnostic dilemmas arise as the full phenotype may not be manifest at the initial assessment. There is a high prevalence of isolated features of Marfan's syndrome in the general population, such as joint hypermobility, mitral valve prolapse, early myopia, and scoliosis, which contributes to the difficulty in making a specific diagnosis of sporadic Marfan's syndrome. Furthermore, genetic heterogeneity must be recognised as many of these skeletal features are found in other connective tissue disorders such as the related condition Beal's congenital contractural arachnodactyly, caused by mutations within the fibrillin gene on chromosome $5,^{30}$ homocysteinuria, and Ehlers-Danlos syndrome.

The detection rate of mutations within the fibrillin gene on chromosome 15 is extremely low (10-20\%), and with one exception, all mutations identified have been unique to a single family. ${ }^{3132}$ This precludes the widespread use of direct mutation analysis for the diagnosis of sporadic Marfan's syndrome. Some 25\% of individuals with Marfan's syndrome will have no family history of the condition and diagnosis will therefore rely on clinical criteria. Children who do not fulfil these criteria but who have at least four skeletal features, particularly a pectus deformity, scoliosis, and limb disproportion in association with tall stature, in the absence of overt cardiovascular or ocular manifestations, should undergo periodic re-evaluation as more diagnostic features may become apparent with increasing age.

We recommend that annual review should be undertaken to assess growth and vertebral column abnormality in children in whom sporadic Marfan's syndrome is suspected on the basis of four or more skeletal characteristics. Annual slit lamp examination to the age of 6 years will detect ectopia lentis in most children. ${ }^{28}$ Thereafter visual acuity testing is appropriate. Yearly echocardiographic examination is recommended even in the absence of overt cardiovascular abnormality as aortic dilatation is clinically silent. By the time that skeletal maturity is attained and the full musculoskeletal phenotype is apparent, most affected individuals will have evidence of cardiovascular abnormality and clinical diagnosis of Marfan's syndrome is simplified. There may still be individuals who will develop cardiovascular features for the first time during adulthood and therefore continued echocardiographic surveillance may be appropriate in a few individuals who do not fulfil the full clinical diagnostic criteria ${ }^{5}$ but who have six or more musculoskeletal features in adulthood.

This project was supported by a Medical Innovation Fund grant.

1 Pyeritz R, McKusick VA. The Marfan syndrome: diagnosis and management. N Engl F Med 1979;300:772-7.

2 Kainulainen K, Pulkkinen L, Savolainen A, Kaitila I, Peltonen L. Location on chromosome 15 of the gene causing Marfan syndrome. N Engl f Med 1990;323:935-9.

3 Dietz HC, Cutting GR, Pyeritz RE. Marfan syndrome caused by a recurrent de novo missense mutation in the fibrillin gene. Nature 1991;352:337-9.

4 Hollister DW, Godfrey M, Sakai LY, Pyeritz R. Immunohistologic abnormalities of the microfibrillar-fiber system in the Marfan syndrome. N Engl F Med 1990;323:152-9.

5 Beighton P, de Paepe A, Danks D, et al. International nosology of heritable disorders of connective tissue, Berlin 1986 . Am f Med Genet 1988;29:581-94.

6 Pereira L, Levran O, Ramirez F, et al. A molecular approach to the stratification of cardiovascular risk in families with Marfan's syndrome. N Engl F Med 1994;331:148-53. 
7 Safarzi M, Tsipouras P, Del Maestro R, et al. A linkage map of 10 loci flanking the Marfan syndrome locus on $15 \mathrm{q}$ results of an in

8 Dietz HC, Pyeritz R, Hall BD, et al. The Marfan syndrome locus: confirmation of assignment to chromosome 15 and identification of tightly linked markers at 15q15-q21.3. Genomics 1991;9:355-61.

9 Sisk H, Zahka K, Pyeritz R. The Marfan syndrome in early childhood: analysis of 15 patients diagnosed at less than 4 years of age. Am F Cardiol 1983;52:353-8.

10 Geva T, Hegesh J, Frand M. The clinical course and echocardiographic features of Marfan's syndrome in childhood. Am F Dis Child 1987;141:1179-82.

11 Morse R, Rochenmacher S, Pyeritz R, et al. Diagnosis and management of infantile Marfan's syndrome. Pediatrics 1990;86:888-95.

12 Phornphutkul C, Rosenthal A, Nadas AS. Cardiac manifestations of Marfan syndrome in infancy and childhood. Cirtations of Marfan syndrom
culation 1973;47:587-96.

13 Tayel S, Kurczynski T, Levine M, et al. Marfanoid children. Am $\mathcal{F}$ Dis Child 1991;145:90-3.

14 El Habbal M. Cardiovascular manifestations of Marfan's syndrome in the young. Am Heart $\mathcal{F} 1992 ; 123: 752-7$.

15 Halpern BL, Char F, Murdoch T, Horton W, McKusick VA. A prospectus on the prevention of aortic rupture in the Marfan syndrome with data on survivorship without treatment. fohns Hopkins Med f 1971;129:123-9.

16 Pyeritz RE. Diagnosis and management of cardiovascular disorders in the Marfan syndrome. F Cardiovasc Med 1980 5:759-69.

17 Gross D, Robinson L, Smith L, Glass N, Rosenberg H, Duvic D Severe perinatal Marfan syndrome. Pediatrics 1989; 84: 84-9.

18 Buntinx I, Willems P, Spitaels S, Van Reempst P, De Paepe A, Durnan J. Neonatal Marfan syndrome with congenital arachnodactyly, flexion contractures, and severe cardiac valve insufficiency. 7 Med Genet 1991;28:267-73.

19 McKusick VA. The Marfan syndrome. Heritable disorders of connective tissue. 4th Ed. St Louis: Mosby, 1972:61-223.

20 Steinberg DA. A simple screening test for the Marfan syndrome. AfR 1966;97:118-24.
21 Walker BA, Murdoch JL. The wrist sign: a useful physical guide to the Marfan syndrome. Arch Intern Med 1970;126: 276-7.

22 Beighton P, Grahame R, Bird H. Hypermobility of joints. 2nd Ed. London: Springer-Verlag, 1989:149-70.

23 Roman MJ, Devereux RB, Kramer-Fox R, O’Loughlin B. Two dimensional echocardiographic aortic root dimensions in children and adults. Am f Cardiol 1989;64:507-12.

24 Collod G, Babron M, Jondeau G, et al. A second locus for Marfan syndrome maps to chromosome 3p24.2-p25. Nat Genet 1994;8:264-8.

25 Kainulainen K, Karttunen L, Puhakka L, Sakai L, Peltonen L. Mutations in the fibrillin gene responsible for dominant ectopia lentis and neonatal Marfan syndrome. Nat Genet 1994;6:64-9.

26 Milewicz D, Duvic M. Severe neonatal Marfan syndrome resulting from a de novo 3-bp insertion into the fibrillin gene on chromosome 15. Am f Hum Genet 1994;54:447-53.

27 Deitz H, Pyeritz R, Puffenberger E, et al. Marfan phenotype variability segregating a missense mutation in the epidermal growth factor-like motif of the fibrillin gene. $\mathcal{F}$ Clin Invest 1992;89:1674-80.

28 Alcorn DM, Maumenee I. The natural history of lens dislocation in children with the Marfan syndrome [abst]. $A m \mathcal{F}$ Hum Genet 1989;45:38.

29 Maumenee.IH. The eye in Marfan syndrome. Trans $\mathrm{Am}$ Ophthalmol Soc 1981;79:684-733.

30 Tsipouras P, Del Mastro R, Sarfazi M, et al. Genetic linkage of the Marfan syndrome, ectopia lentis, and congenital contractural arachnodactyly to the fibrillin genes on chromosomes 15 and 5. N Engl F Med 1992;326:905-9.

31 Deitz HC, McIntosh I, Sakai L, et al. Four novel FBN1 mutations: significance for mutant transcript level and EGF-like domain calcium binding in the pathogenesis of Marfan's syndrome. Genomics 1993;17:468-75.

32 Haywood J, Rae A, Porteous M, Logie L, Brock D. Two novel mutations and a neutral polymorphism in the EGFlike domains of the fibrillin gene (FBN1): SSCP screening of exons15-21 in Marfan syndrome patients. Hum Mol Genet 1994;3:373-5. 